\title{
When the Coronary Anomaly Saves Your Life: a Very Rare Ischemic Coronary Presentation - Our Cardiac Catheterization Laboratory Experience and New Way of Treatment
}

\author{
Piraino. $\mathrm{D}^{1 *}$, Arrotti.S ${ }^{2}$, Bracale.U.M $\mathbf{M}^{3}$, Dendramis.G' ${ }^{2}$, Paleologo.C ${ }^{2}$, Teresi.G ${ }^{2}$, Buccheri. ${ }^{1}$, \\ Carella. $\mathbf{M}^{1}$, Manzullo. ${ }^{2}$, Andolina. $\mathbf{G}^{1}$ and Assennato. $\mathrm{P}^{2}$ \\ 1Section of Interventional Cardiology and Hemodynamics, A.O.U.P. Paolo Giaccone, Palermo, Italy \\ 2Section of Intensive Coronary Care Unit, A.O.U.P. Paolo Giaccone, Palermo, Italy \\ 3Vascular and Endovascular Surgery Unit, University Federico II of Naples
}

Received: November 18, 2014; Accepted: February 19, 2015; Published: February 26, 2015

*Corresponding author: Davide Piraino, Section of Interventional Cardiology and Hemodynamics, A.O.U.P. Paolo Giaccone, Palermo, Italy Tel: 389346-7721; E-mail: pirainod@libero.it

\begin{abstract}
Left main coronary artery acute occlusion is a dramatic event, with a higher risk of sudden death, due to hemodynamic instability or malignant arrhythmia. In these, patients appear to be crucial on immediate and prompt revascularization to prevent fatal outcome. We report a very rare ischemic pattern, with the association of left main coronary artery acute occlusion and a coronary anomaly which contributes to save patient life.
\end{abstract}

Keywords: Left main coronary artery; Percutaneous coronary intervention; Intra aortic balloon pump; Myocardial infarction; Drug eluting stent

\section{Abbreviations}

BMS: Bare metal stent; BVS: Bioresorbable Vascular Scaffold; CAA: Coronary Artery Anomaly; CABG: Coronary Artery Bypasses Graft; CASS: Coronary Artery Surgery Study; DES: Drug Eluting Stent; ECG: Electrocardiogram; EPCs: Circulating Endothelial Progenitor Cells; IABP: Intra Aortic Balloon Pump; ISR: In-Stent Restenosis; IVUS: Intravascular Ultra Sound; LAD: Left Anterior Descending; LCX: Left Circumflex Artery; LMCA: Left Main Coronary Artery; MLA: Minimal Lumen Area; PCI: Percutaneous Coronary Intervention; RCA: Right Coronary Artery; TIMI: Thrombolysis in Myocardial Infarction.

\section{Introduction}

Left Main Coronary Artery (LMCA) acute occlusion is a rare event, with high risk of sudden death. This emergency requires immediate revascularization to avert fatal outcome: this would explain because the literature about LMCA acute occlusion is incomplete: majority of patients do not arrive on time for an attempted revascularization.
The chances of survival may be increased, if between LMCA occlusion and patient's life are interposed with an unexpected coronary anomaly.

Coronary artery anomalies are rare and in literature the reported prevalence is about $0.6 \%$ to $1.3 \%$ [1] in angiographic diagnostic procedure and $0.3 \%$ in autopsy series, because many anomalies don't give symptoms during the patient's life (only $20 \%$ of patients have symptoms during their life) [2] and their reply is an incidental finding, especially when LMCA is involved, as in our case report, and a very rare anomalous origin of the Circumflex Artery(LCX) from Right Coronary Artery (RCA) plays an important role.

\section{Case Report}

A-42-year-old cigarette smoking man with chest pain arising for about two hours was admitted to our hospital. No previous cardiac events are reported in his past history. Electrocardiogram (ECG) showed (Figure 1) left bundle branch block (not presented in previous ECGs) and blood test indicated a peak of myocardial necrosis index: cTnI $80 \mathrm{ng} / \mathrm{ml}$. Patient's clinical condition appeared immediately critical, due to the presence of cardiogenic shock, with severe metabolic acidosis, oligoanuria and immediate intubation necessity. Echocardiogram revealed: extended akinesia of anterolateral wall, interventricular septum and apex and ejection fraction $30 \%$.

We performed coronary angiography: LMCA acute thrombotic occlusion (without collateral flow from the RCA) and anomalous origin of LCX from RCA (Figures $2 \mathrm{~A}$ and $\mathrm{B}$ ), both without stenosis, so we placed an intra aortic balloon pump (IABP) and proceeded to mechanical thrombus aspiration and Drug- Eluting Stent (DES) implantation (Xience $3 \times 15 \mathrm{~mm}$ ) between the LMCA-Left Anterior Descending Artery (LAD) (Figure $2 \mathrm{C}$ ). After three days, stabilization of hemodynamic status, intubation and hemodynamic support by IABP were stopped. 
The patient was discharged after 25 days in good clinical condition and without residual angina symptoms. ECG at discharge is shown in Figure 3. Six-months follow up echocardiography showed: slight improvement of systolic function (EF 35\%) with persistent apex, anterolateral wall and interventricular septum akinesia. At the end of 1 year follow up, we re-evaluated the DES implantation in LMCA-LAD by Intravascular Ultra Sound (IVUS) images, to verify the real risk of In-Stent Restenosis (ISR). We have seen an optimal stent apposition with a Minimal Lumen Area (MLA) of 10, $35 \mathrm{~mm}^{2}$ (Figure 4), significantly higher than the limit value for LMCA's significant stenosis $\left(6 \mathrm{~mm}^{2}\right)$ [3].

\section{Discussion}

The equation LMCA occlusion and fatal outcome, due to profound hemodynamic instability or malignant arrhythmia, is almost the rule. The reported incidence of LMCA total occlusion events coming in cathlab is about $0.4 \%-0.5 \%$ [4]. In our experience, over more than 1500 coronary angiography performed every year, only $0.2 \%$ of Percutaneous Coronary Interventions (PCI) show an acute LMCA occlusion.

In literature, worse prognosis is reported when the patients are already in cardiogenic shock and there is not collateral circulation from RCA. Sakai et al. [5] found a higher hospital mortality and lower successful PCI procedure, in patients with cardiogenic shock compared to non shock patients and without a collateral circulation from RCA (47.1\% versus $95.2 \%, p=0.0008)$.

In this patients appear to be crucial for an immediate and prompt revascularization to prevent fatal outcome. In the past, surgical revascularization has always been the preferred

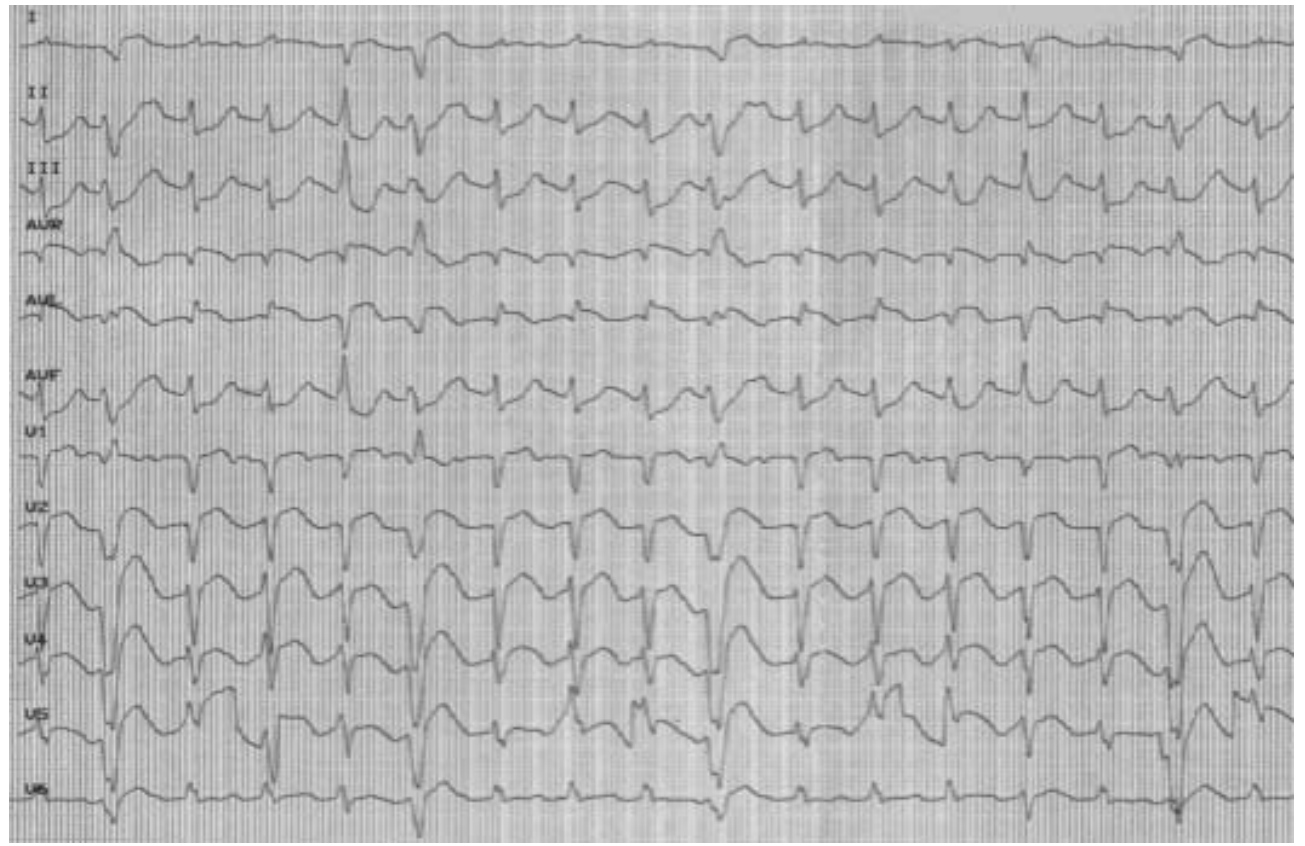

Figure 1: ECG at admission.

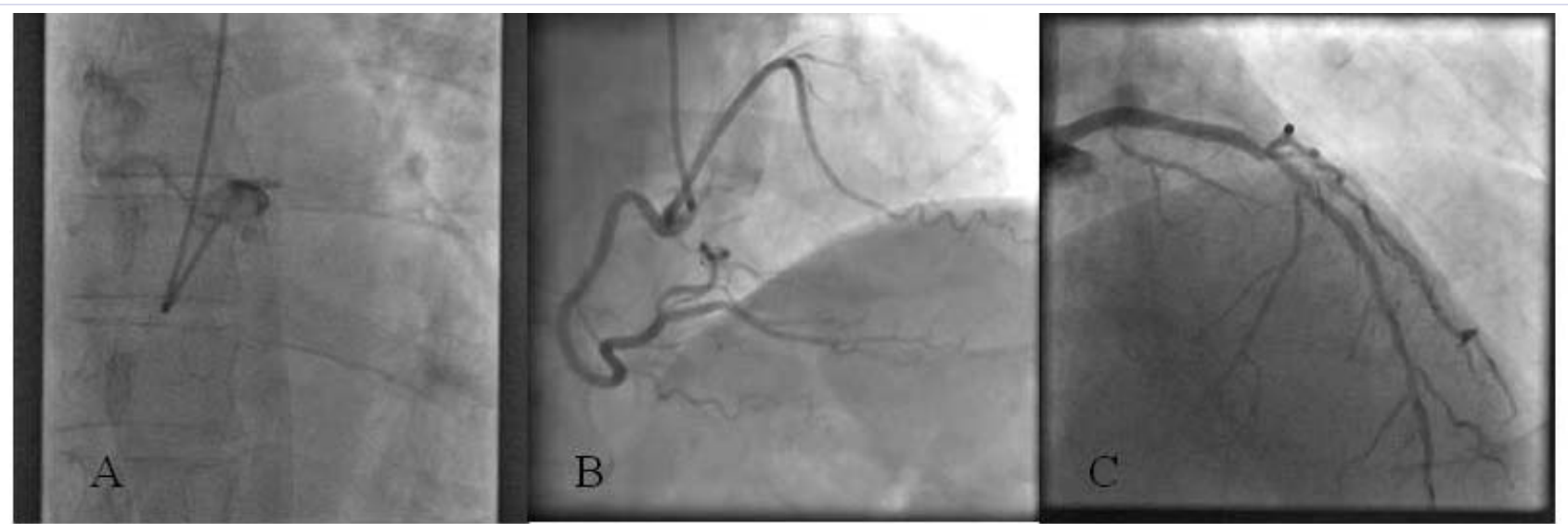

Figure 2: A: LMCA occlusion in right cranial view, B: RCA and anomalous LCX in left oblique view, C: LMCA after DES implantation in right cranial view.

Citation: Piraino D, Arrotti S, Bracale UM, Dendramis G, Paleologo C, et al. (2015) When the Coronary Anomaly Saves Your Life: a Very Rare Ischemic Coronary Presentation - Our Cardiac Catheterization Laboratory Experience and New Way of Treatment. J Clin 

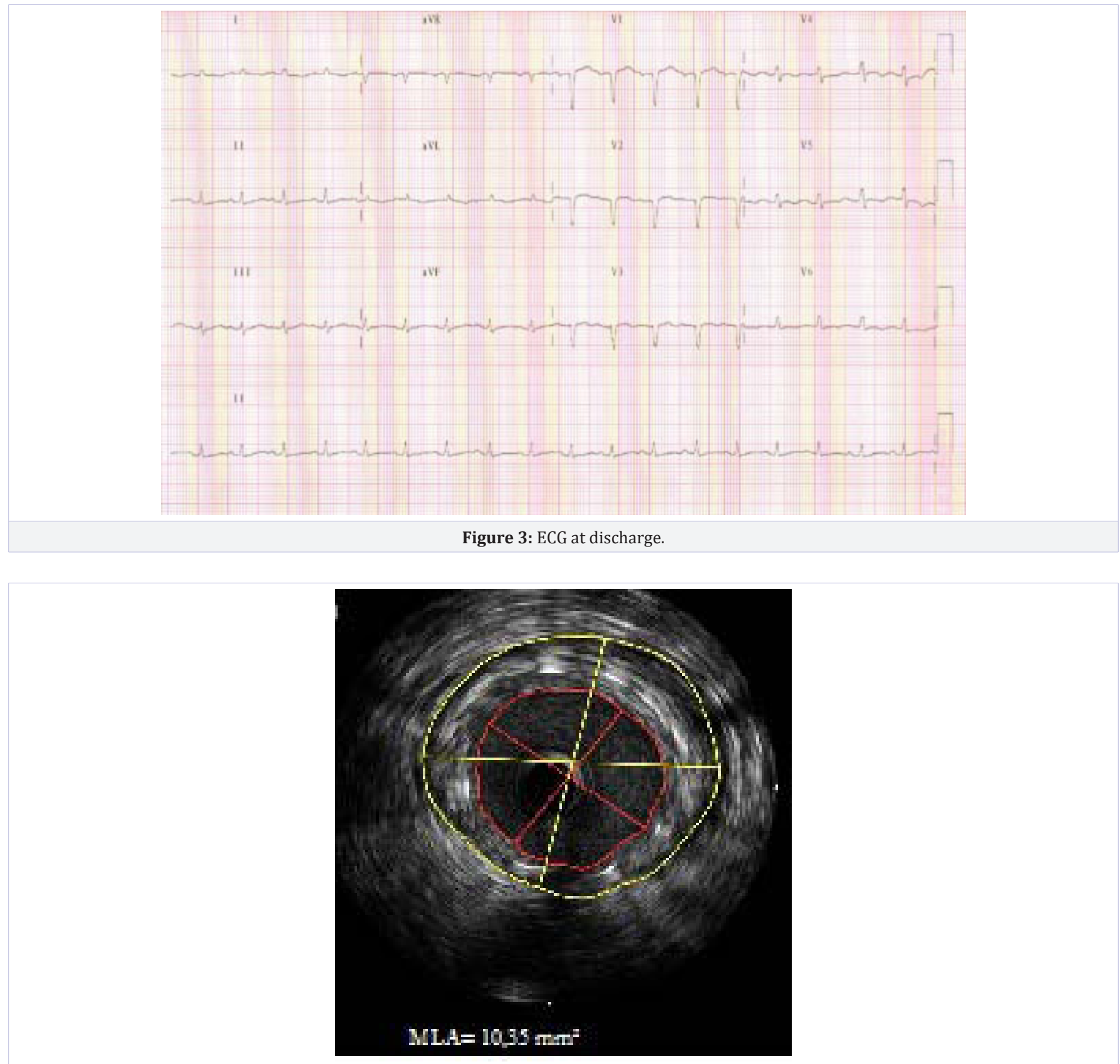

Figure 4: IVUS image after 1 year DES implantation on LMCA.

technique for this type of patients, resulting with this approach from the Coronary Artery Surgery Study (CASS) registry: Coronary Artery Bypass Graft (CABG) showed a survival benefit compared to medical therapy or PCI. However in recent years, with the advent of DES and improvement of revascularization technique this site so important as the LMCA, PCI carved out an important role in the LMCA disease, unimaginable until few years ago.

Recent guidelines on myocardial revascularization [6] indicate $\mathrm{PCI}$ and $\mathrm{CABG}$ as equivalent technique of revascularization in patients with LMCA disease and syntax score $\leq 22$, preferring surgical revascularization in case of multiple vessels involvement (higher syntax score).

These data underscore the differences between the two methods of revascularization in elective LMCA disease, but until now, in literature there are no large randomized trials which give an answer to the appropriate management during acute thrombotic LMCA occlusion. During this catastrophic event, where time factor plays the most important role in patient survival, an immediate revascularization may increase the chances of life, suggesting emergency PCI as the first choice. 
Patel et al [7] in the British Cardiovascular Intervention Society database analyzed unprotected LMCA occlusion treated with primary PCI vs. emergency patients treated with nonocclusive LMCA disease, 1 -year (52.8\% vs. $32.4 \%$; $p<0.001)$, and 3 -year mortality $(73.9 \%$ vs. $52.3 \%, p<0.001)$ rates were higher in patients with unprotected LMCA occlusion, compared with patients presenting with a patent LMCA, and were significantly influenced by the presence of cardiogenic shock

PCI has an advantage offering rapid reperfusion to critically ill patients, often those with very high risk for surgical revascularization, with acceptable short- and long-term outcomes, reducing the risk of ISR and stent thrombosis over the last years, thanks to the improvement of revascularization techniques and available devices.

Among the total cases of LMCA acute thrombotic occlusion of our catheterization laboratory, we describe this case report because it offers two meditation points. The patient was in cardiogenic shock, severe ejection fraction reduction, Killip class IV and absence of collateral circulation from RCA, so we had a high risk patient and not enough time to opt for a surgical revascularization. But in our case report, in addition to time factor, the other discriminating factor which increases the chances of patient's survival was coronary anomaly: an anomalous LMCA, with LCX originating from RCA. An anomalous origin of the LCX from the right sinus of Valsalva with separate ostium of RCA is a well recognized anatomical variant, albeit rare (prevalence of $0.67 \%$ ), [8] as well as even rarer when LCX originates from the proximal segment of RCA, as in our clinical case (prevalence $0.08 \%$ [1]. The recognition of this anomaly coronary is fundamental, as previously reported in literature [9$10]$ both for a suitable treatment, in case of stenosing pathology, both for a correct evaluation of myocardial damage extension during an acute coronary syndrome.

The associated symptoms are infrequent (generally about $80 \%$ of patients with coronary anomalies are asymptomatic) [2], therefore, as in our case, it's often an incidental finding. When the symptoms are present, they are extremely variable and nonspecific for a coronary anomaly hypothesis, including chest pain, dyspnea, arrhythmias, until clearer and more important symptoms as syncope, myocardial infarction or sudden death. These symptoms concern not benign coronary anomalies requiring revascularization, regardless of the presence of atherosclerotic disease because the risk of adverse event seems to be related to abnormal course between aorta and pulmonary artery, with a high risk, in course of physical effort, the distension of these two vessel could result in compression or kinking of the abnormal course of anomaly coronary.

This is also explained because in normal circumstances cardiac examination and ECG does not clarify the problem, while a stress test could provide indirect information, not on the presence of coronary artery anomaly, but about the possible ischemia associated with it. The incidence of LCX arising from the RCA is generally believed to be of no clinical significance and it does not predispose LCX to a higher incidence of obstructive disease, due to a dorsal course the left ventricle [11]. When there is an occasional coronary anomaly finding ,as LCX arising from the proximal segment of the RCA, with atherosclerotic disease to be treated, we must consider the difficulties which may face in the revascularization process: it's necessary to use a catheter which gives adequate support to RCA ostium, as well as an extra-support guide-wire because arising from the RCA, the course of LCX could be more torturous worsening the deliverability and track ability of devices to reach the site of revascularization.

In this situation, we find two very rare situations which are not previously reported in literature: LMCA acute occlusion associated to a rare coronary anomaly. Not only the time factor played a benign role in this situation, but also the very rare coronary artery anomaly, with a separate LCX, has allowed us to save the patient's life. Coronary anomaly is not always a negative congenital disorder.

\section{Conclusion}

Acute LMCA occlusion is a rare and catastrophic event, but sometimes a particularly unexpected help could come from an occasional finding of a coronary anomaly, as a LCX arising from the proximal RCA. This coronary anomaly may be given a chance of survival to the patient, giving us enough time to treat the acute LMCA occlusion with an adequate and prompt revascularization.

\section{New Devices: An Extra Weapon Available for PCI}

Over the last years, unthinkable progress have been done on treatment of LMCA disease, thanks to the improvement of the techniques of revascularization and available device that have progressively reduced the risk of adverse events in terms of ISR and stent thrombosis, especially in a place which is so important as LMCA and in a particular situation as an ischemic event. In fact, in a hard situation as STEMI, the risk of ISR is higher because the interventional cardiologists don't have the real vessel size due to inflammation vasoconstriction, spasm and thrombotic stratification and this is the reason for a higher rate of stent thrombosis too [12].

These new types of stents contribute further reduction risk:

\section{Bioresoursable Vascular Scaffolds (BVS)}

Bioresorbable, consists of a frame poly L-lactide component with a full and complete resorption over the next two years reduces the risk of restenosis and late thrombosis due to the absence of metal struts. In the absence of metallic components, it compromises to accept an adequate radial force with greater thickness struts that can make it less easy to handle in terms of deliverability, tractability and ability to dilate the lesion, especially if calcified.

Two studies have recently been published, reporting the feasibility and safety to Absorb BVS implantation specifically in STEMI patients [13]. With very limited data on clinical use of the BVS in acute patients, several aspects need to be carefully evaluated; among them was the effective incidence of scaffold thrombosis. 


\section{Mesh-covered stent}

Recently this device was introduced to reduce distal embolisation of debris from the thrombotic occlusion lesion during an acute ischemic event and stent deployment. This debris promote microvascular dysfunction after primary PCI with an increase infarct size and worse follow up in terms of major adverse cardiac events. This Bare Metal Stent (BMS) covered with a polyethylene terephthalate micronet mesh was introduced to trap thrombotic material between the stent and the arterial wall, thus theoretically reducing the risk of no-reflow or slow-flow due to distal embolisation.

A randomized trial has been performed to compare the rate of complete ( $\geq 70 \%$ ) ST-segment resolution measured at 60 to $90 \mathrm{~min}$ post-procedure, between mesh-covered stents and standard stents [14]. This endpoint was significantly improved in patients treated with mesh-covered stents (57.8\% vs. $44.7 \%$; $p$ $=0.008$ ), also with a superior rate of Thrombolysis in Myocardial Infarction (TIMI) 3 flow ( $91.7 \%$ vs. $82.9 \%, p=0.006$ ) with an equivalent myocardial blush grade 2 or $3(83.9 \%$ vs. $84.7 \%$, $p=0.81$ ) compared with standard stents. Although a meshcovered stent in STEMI could have interesting implications especially in relation to the reduction of thrombus dislodgement and embolisation, being a BMS and because of the presence of permanent polyethylene micronet mesh suggesting the need for careful assessment of restenosis rate.

\section{Self expanding stents}

This stent have a memory shape, so the stent self-deploys gently and atraumatically in the vessel without the need for a deployment balloon. Stent platform is self-apposing and conforms to the vessel profile in which it is deployed due to its elastic properties and very thin stent struts, allowing for a more anatomical fit. The stent expands until it reaches the vessel wall, and exerts a continuous, gentle outward force onto the vessel walls, such that any positive remodeling over time would be accommodated by the stent through subsequent expansion.

This characteristic is fundamental during an acute ischemic event, because we don't have the real idea of the vessel size (due to vessel inflammation vasoconstriction, thrombotic stratification, spasm): delicate apposition does not increase vascular damage in this phase, without an increased risk of embolization and nonreflow phenomenon, acquiring its real volume in the following weeks, when passed to the acute phase, the vessel has regained its real size [15].

\section{Dual Therapy Stent (COMBO stent)}

A new very promising stent, in the elective procedures both in course of acute ischemic event. It is a unique stent because its property of dual therapy combining low-dose Sirolimus delivery from an alumina biodegradable polymer matrix along with a covalently bound anti-CD34 antibody layer in combination with device designed for control of neointimal proliferation as well as to promote vessel healing through a homogeneous coverage.

The role of these antibodies is to capture circulating
Endothelial Progenitor Cells (EPCs) from the blood flow in short time with an adequate coverage of metallic struts and without an excess of neointima. In this way COMBO stent reduces the risk of ISR and stent thrombosis, without being present in metallic uncovered struts thereby obtaining an optimal control of the formation of neointima. These features turn out to be fundamental in a site so difficult to treat as the LMCA is elective but more especially used in course of an acute ischemic event. In the literature it is reported that, during a STEMI, the share of circulating EPCs increases considerably $(167 \%$ more than the control group), emphasizing on the course of an acute ischemic event, this type of stent plays a fundamental role [16]. The REMEDEE OCT trial is designed to compare the early vascular healing of the Combo Stent and the available everolimus-eluting stents in patients with acute Coronary Syndrome (STEMI or NSTEMI). The use of these stents in course of an acute ischemic event seems to be particularly suitable and this study will give the information needed to understand the reliability during NSTEMI or STEMI [17].

\section{Bibliography}

1. Ahmet Y, Baris O,Tezcan P, Caner A, Ayhan O, Bulent M, et al. Prevalence of Coronary Artery Anomalies in 12,457 Adult Patients Who Underwent Coronary Angiography. Clin. Cardiol.2010; 33(12): E60-E64. D0I:10.1002/clc.20588.

2. Datta J, White CS, Gilkeson RC, Cristopher A. Meyer, Sarita. K, et al. Anomalous coronary arteries in adults. depiction at multi-detector row CT angiography. Radiology. 2005; 235(3): 812-818.

3. De la Torre Hernandez JM, Hernández F, Alfonso F, Jose R. Rumoroso, Ramon Lopez-Palop, Mario.S, et al. Prospective application of Pre-defined intravascular ultrasound criteria for assessment of intermediate left main coronary artery lesions results from the multicenter LITRO study. J Am Coll Cardiol 2011; 58 (4): 351-358. doi:10.1016/j.jacc.2011.02.064.

4. Neri R, Migliorini A, Moschi G, Renato. M, Emilio. V.D, David.A. Percutaneous reperfusion of left main coronary disease complicated by acute myocardial infarction. Catheter Cardiovascular Interv. 2002; 56:31-4. DOI: 10.1002/ccd.10168.

5. Sakai K, Nakagawa Y, Kimura T, Kimura T, Ando K, Yokoi H, Iwabuchi $\mathrm{M}$, et al. Primary angioplasty of unprotected left main coronary artery for acute anterolateral myocardial infarction. J Invasive Cardiol 2004; 16: 621-5.

6. Windecker S, Kolh P, Alfonso F, Fernando A, Jean-Philippe Collet, Jochen C, Volkmar F, et al. 2014 ESC/EACTS Guidelines on myocardial revascularization. The Task Force on Myocardial Revascularization of the European Society of Cardiology (ESC) and the European Association for Cardio-Thoracic Surgery (EACTS). European Heart Journal (2014) 35, 2541-2619.

7. Patel N, De Maria GL, Kassimis G, George K, Kazem R, Derrick B, et al. Outcomes after Emergency Percutaneous Coronary Intervention in Patients with Unprotected Left Main Stem Occlusion: The BCIS National Audit of Percutaneous Coronary Intervention 6-Year Experience. JACC Cardiovasc Interv. 2014 Sep; 7(9): 969-80. doi:10.1016/j.jcin.2014.04.011.

8. Angelini P, Villason S, Chan AV and Diez JG. Normal and anomalous coronary arteries in humans. In: Angelini P, ed. Coronary artery anomalies: a comprehensive approach. Philadelphia,PA: Lippincott Williams \& Wilkins, 1999: 27-150. 
9. Dursun Dursunoðlu, Güllü Özalp, Özgür Tapköylü and Ender Semiz. Anomalous origin of the left circumflex coronary artery: A case report. Exp Clin Cardiol 2007; 12(4): 207-208.

10. Sotiris C Plastiras, Ourania S Kampessi, Maria Gotzamanidou and Panayiotis Kastanis. Anomalous origin of the left circumflex artery from the right coronary artery: a case report. Cases Journal 2008, 1(1): 336.

11. Budoff MJ, Ahmed V, Gul KM, Mao SS and Gopal A. Coronary anomalies by cardiac computed tomographic angiography. Clin Cardiol. 2006; 29: 489-493.

12. Kristensen S.L, Galloe AM, Thuesen L, Kelbæk H, Thayssen P, Havndrup 0 . et al. Stent thrombosis is the primary cause of ST-segment elevation myocardial infarction following coronary stent implantation: a five year follow up of the SORT OUT II study. PLoS One. 2014; 9(11): 113399. doi: 10.1371/journal.pone.0113399.

13. Diletti R, Karanasos A, Muramatsu T, Nakatani S, Van Mieghem NM, Onuma Y. et al. Everolimus-eluting bioresorbable vascular scaffolds for treatment of patients presenting with ST-segment elevation myocardial infarction: BVS STEMI first study. Eur Heart J. 2014; 35: 777-86. doi: 10.1093/eurheartj/eht546.
14. Kitabata $\mathrm{H}$, Kubo T, Ishibashi $\mathrm{K}$, Komukai $\mathrm{K}$, Tanimoto $\mathrm{T}$, Ino $\mathrm{Y}$, et al. Prognostic value of microvascular resistance index immediately after primary percutaneous coronary intervention on left ventricular remodeling in patients with reperfused anterior acute ST-segment elevation myocardial infarction. JACC Cardiovasc Interv. 2013; 6: 1046-54. doi: 10.1016/j.jcin.2013.05.014.

15. Van Geuns RJ, Tamburino C, Fajadet J, Vrolix M, Witzenbichler B, Eeckhout E, et al. Self-expanding versus balloon-expandable stents in acute myocardial infarction: results from the APPOSITION II study: self-expanding stents in ST-segment elevation myocardial infarction. JACC Cardiovasc Interv. 2012; 5: 1209-19. doi: 10.1016/j. jcin.2012.08.016.

16. Leone AM, Rutella S, Bonanno G, Abbate A, Rebuzzi AG, Giovannini S, et al. Mobilization of bone marrow-derived stem cells after myocardial infarction and left ventricular function. Eur Heart J. 2005 Jun; 26(12): 1196-204.

17. Woudstra P, de Winter RJ, Beijk MA. Next-generation DES: the COMBO dual therapy stent with Genous endothelial progenitor capturing technology and an abluminal Sirolimus matrix. Expert Rev Med Devices. 2014 Mar; 11(2): 121-35. doi: 10.1586/17434440.2014.882046. 